\title{
The continuing story of two-photon exchange: results from the OLYMPUS experiment
}

\author{
Axel Schmidt ${ }^{* \dagger}$ \\ Massachusetts Institute of Technology \\ E-mail: schmidta@mit.edu
}

Over the past two decades, a discrepancy has emerged between two different techniques for measuring the proton's electromagnetic form factors. Unpolarized electron-proton cross section measurements paint a picture of the proton's internal structure that is incompatible with measurements from polarization transfer experiments. The leading hypothesis is that the discrepancy is caused by a typically neglected radiative correction, hard two-photon exchange (TPE), which would affect the two measurement techniques in different ways. There is no model independent way to calculate hard TPE, but it can be measured experimentally by looking for an asymmetry between the positron-proton and electron-proton elastic cross sections. Three recent experiments have attempted to quantify this asymmetry, and, just last month, the third of these, called OLYMPUS, released its results [1]. The OLYMPUS experiment collected data in 2012 at DESY, alternating between $2 \mathrm{GeV}$ electron and positron beams, directed through a hydrogen gas target. The scattered lepton and recoiling proton were detected in coincidence with a large acceptance toroidal spectrometer. The relative luminosity between the two beam species was monitored with three independent systems, and the results comprise 3 inverse fb of integrated luminosity, exceeding by a factor of three the other two TPE experiments combined. In this talk, the case for the TPE hypothesis will be presented, the OLYMPUS experiment will be described, and the results of all three experiments will be compared.

55th International Winter Meeting on Nuclear Physics

23-27 January, 2017

Bormio, Italy

* Speaker.

${ }^{\dagger}$ for the OLYMPUS Collaboration 


\section{Introduction}

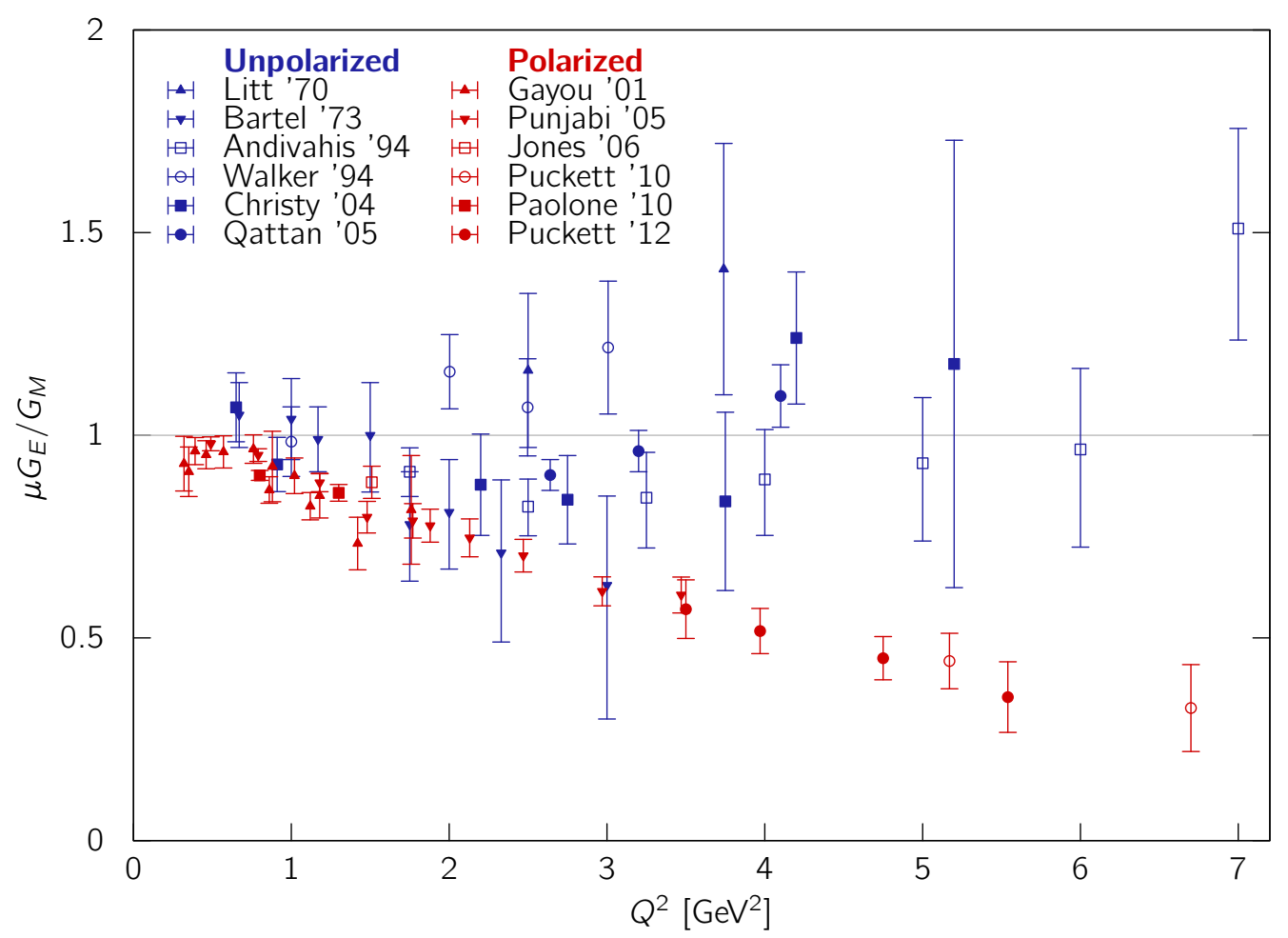

Figure 1: Measurements of the proton's form factor ratio $\mu_{p} G_{E} / G_{M}$ extracted from polarization asymmetries [2-7] do not agree with those from unpolarized Rosenbluth separation [8-13].

Over the past few decades, a discrepancy emerged between two different techniques for measuring the proton's elastic electromagnetic form factors. These form factors, $G_{E}\left(Q^{2}\right)$ and $G_{M}\left(Q^{2}\right)$, describe the proton's distributions of charge and current respectively as functions of the momentum transfer scale $Q^{2}$. Since the 1950s, the proton's form factors were extracted from unpolarized electron-scattering cross sections using Rosenbluth Separation [14]. The advent of high-quality polarized electron beams made it possible to extract the ratio $G_{E} / G_{M}$ from double spin asymmetries [15]. The two techniques have produced discrepant results, as can be seen in the representative sample of world data shown in figure 1. Until this discrepancy is understood, it is difficult to have complete confidence in our understanding of the proton's electromagnetic structure.

The leading hypothesis for the cause of the discrepancy is hard two-photon exchange (TPE), which can be measured by taking the ratio of positron-proton to electron-proton elastic scattering cross sections. Three recent experiments have measured this ratio to test the TPE hypothesis, and with the recent release of the OLYMPUS results [1], all three have published their findings. The results are not strongly conclusive. OLYMPUS measured the ratio to be smaller than many predictions, but the results are still consistent with hard TPE being the cause of the discrepancy.

2. Why measure $\sigma_{e^{+}} / \sigma_{e^{-}}$?

The leading hypothesis for the cause of the proton form factor discrepancy is that there is 
a non-negligible contribution from hard TPE, a radiative correction that is neglected in standard radiative correction prescriptions [16,17]. If hard TPE were properly accounted for, it is possible that the polarization asymmetry and Rosenbluth separation measurements would actually be consistent with each other. Unfortunately, there is no model independent method for calculating hard TPE, though there are numerous model-dependent approaches, such as using hadronic intermediate states [18-20], dispersion relations [21-23], generalized parton distributions [24], and phenomenological fits [25-27]. Many predictions suggest that the inclusion of hard TPE would resolve the form factor discrepancy, while some disagree [28, 29].

The contribution from hard TPE can be determined experimentally through a measurement of $R_{2 \gamma}$, the cross section ratio of elastic positron-proton scattering to elastic electron-proton scattering (after standard radiative effects have been accounted for):

$$
R_{2 \gamma} \equiv \frac{\sigma_{e^{+} p \rightarrow e^{+} p}}{\sigma_{e^{-} p \rightarrow e^{-} p}}
$$

At leading order, this ratio is equal to unity. The next to leading order term is an interference between one- and two-photon exchange. This interference term changes sign when switching between electron and positron scattering, so that $R_{2 \gamma}$ can be written:

$$
R_{2 \gamma}=1+\frac{4 \operatorname{Re}\left[\mathscr{M}_{2 \gamma}^{\dagger} \mathscr{M}_{1 \gamma}\right]}{\left|\mathscr{M}_{1 \gamma}\right|^{2}}+\mathscr{O}\left(\alpha^{4}\right) .
$$

Deviations in $R_{2 \gamma}$ from unity indicate a contribution from hard TPE.

In addition to its magnitude, the kinematic dependence of $R_{2 \gamma}$ is also relevant. For TPE to help resolve the discrepancy, $R_{2 \gamma}$ should increase as $\varepsilon$, the virtual photon polarization parameter, decreases and as $Q^{2}$ increases.

\section{Experiments}

Three recent experiments endeavored to measure $R_{2 \gamma}$. In addition to OLYMPUS, an experiment at the VEPP-3 storage ring in Novosibirsk, Russia, collected data in 2009 and 2012, and an experiment using the CLAS spectrometer in Hall B at Jefferson Lab, USA, collected data in 2011. Summaries of these experiments are presented below.

\subsection{The OLYMPUS Experiment}

The OLYMPUS experiment [30] was conducted in 2012 at the DORIS storage ring at DESY, Hamburg, Germany. DORIS was modified so as to facilitate the acceleration and storage of electrons or positrons within the ring. During data collection, the beam species was alternated approximately once per day.

The DORIS beam was directed through a windowless hydrogen gas target that was internal to the ring vacuum [31]. A differential pumping system of six turbomolecular vacuum pumps was necessary to continuously remove the hydrogen gas that flowed from the ends of the target and prevent it from spoiling the ring vacuum.

The target was surrounded by a large-acceptance toroidal magnetic spectrometer. An illustration of the spectrometer is shown in figure 2. The spectrometer was previously used in the BLAST 


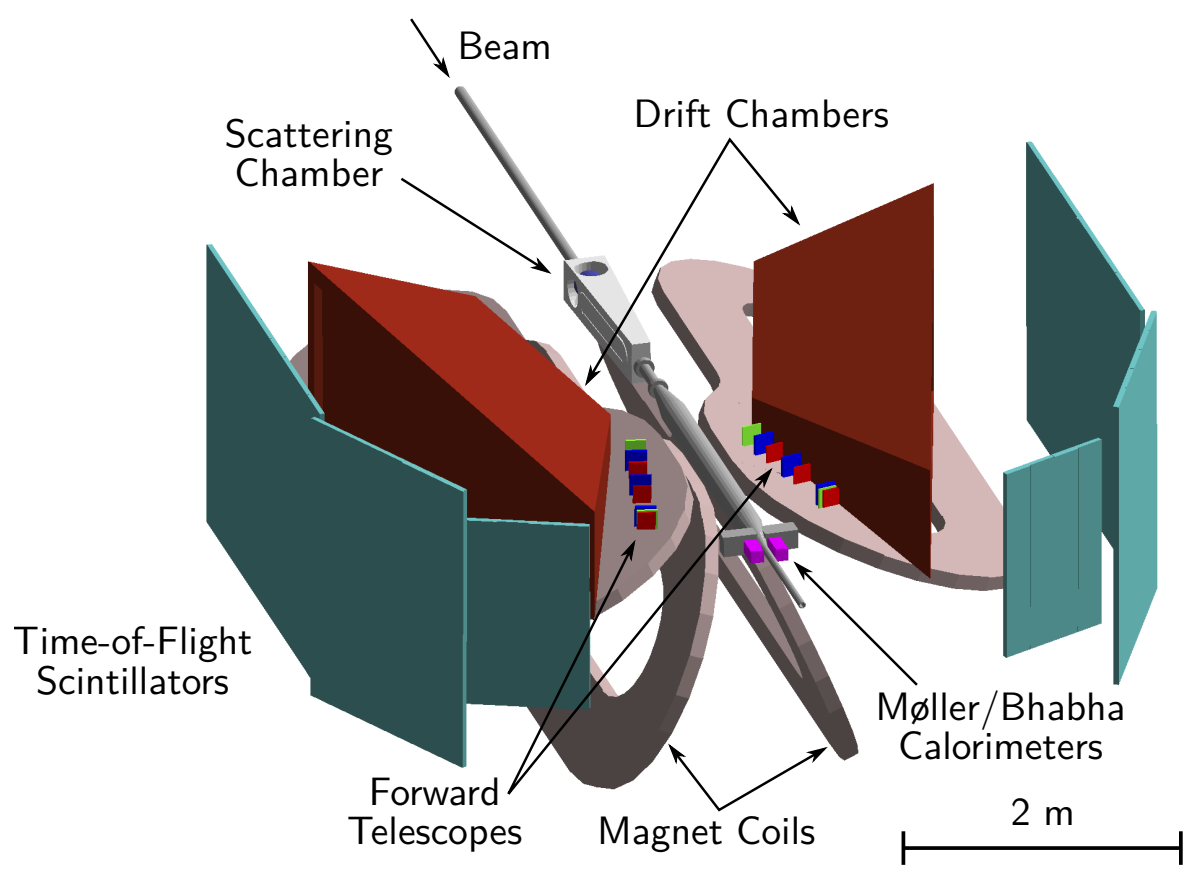

Figure 2: The OLYMPUS spectrometer was formerly used in the BLAST experiment.

experiment [32], which ran from 2001-2005 at MIT-Bates. The spectrometer had two instrumented sectors, one to the left and the other to the right of the target chamber. Layers of drift chambers were used for tracking charged particles, and walls of scintillator were used for time-of-flight measurements and triggering. Elastic $e p$ events were identified by a coincidence detection of the scattered lepton in one sector and the recoiling proton in the other.

In order to extract $R_{2 \gamma}$, it was crucial to determine the relative luminosity between the electron and positron data sets. For this purpose, three independent luminosity monitoring systems were employed. By recording the instantaneous beam current as well as the target flow, the slow control system could be used to reconstruct the relative luminosity to within a few percent. A pair of tracking telescopes were deployed at forward angles to monitor the rate of elastic ep scattering in kinematics where TPE effects were thought to be small. A pair of calorimeters were positioned next to the downstream beamline to measure the rate of symmetric Møller and Bhabha scattering [33].

\subsection{Experiment at VEPP-3}

The experiment at VEPP-3 was similar to OLYMPUS in that it used alternating stored beams of electrons and positrons [34]. However, elastic ep events were detected in a non-magnetic detector. This came with the advantage of having identical acceptances for detecting electrons and positrons. However, without a magnetic field, no momentum analysis of the detected particles was possible. The rejection of inelastic background relied on energy deposition measurements in CsI, $\mathrm{NaI}$, and plastic scintillator detectors.

The relative luminosity between electron and positron modes was also a concern for the VEPP3 experiment. The luminosity was normalized to the forward elastic scattering point, at kinematics 
where TPE effects are expected to be small. Thus, the VEPP-3 measurement of $R_{2 \gamma}$ is relative to $R_{2 \gamma}$ at their luminosity normalization point (LNP).

\subsection{CLAS Two-Photon Experiment}

The CLAS Two-Photon Experiment [35-37] used a teritiary beam of electron/positron pairs. The Hall B secondary photon beam was directed on gold converter foil to create electron and positron pairs, which in turn were directed through a magnetic chicane so that the unconverted photons could be blocked. By combining measurements taken in both chicane polarities, the luminosity of electron and positron data sets could be guaranteed to be equal. However, given the tertiary beam, the initial pre-scatter energy of the lepton in each event was unknown and had to be reconstructed. Scattered leptons and recoiling protons were detected in the CLAS spectrometer, a toroidal magnetic spectrometer with nearly $4 \pi$ coverage.

\section{Results}

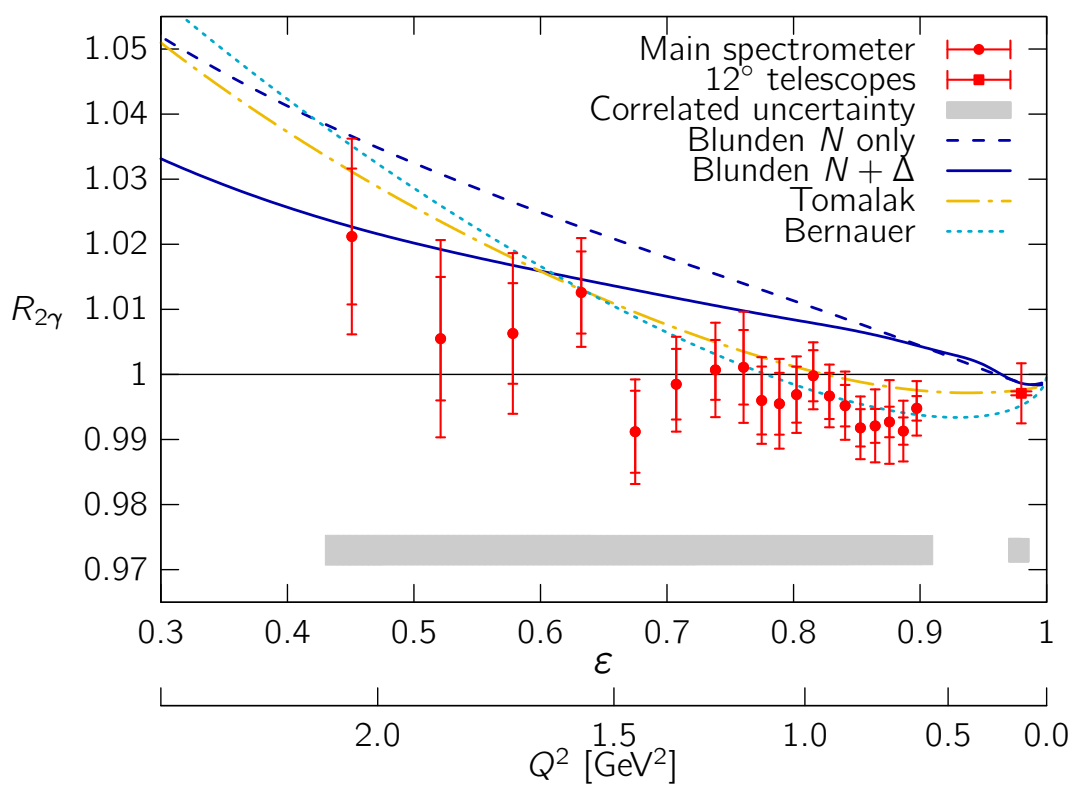

Figure 3: OLYMPUS measured $R_{2 \gamma}$ to be somewhat lower than theoretical calculations by Blunden et al. [23], and Tomalak et al. [22], and the phenomenological prediction by Bernauer et al. [27].

The results of the OLYMPUS experiment [1] are shown in figure 3, along with three predictions for $R_{2 \gamma}$. The inner error bars show the statistical uncertainty, while the outer error bars show the statistical and uncorrelated systematic uncertainties added in quadrature. The grey band below the data shows the $1 \sigma$ correlated uncertainty, though the correlations are more complicated than a simple scale factor, and will be described in a future article. The predictions from Blunden et al. use a dispersive approach to calculate the TPE diagrams with hadronic propagators; the $N$-only prediction assumes only a nucleon propagator only, while $N+\Delta$ assumes the coherent sum of nucleon and $\Delta$ propagators [23]. The calculation of Tomalak et al. uses subtracted dispersion relations to evaluate the TPE diagrams [22]. The curve shown corresponds to a subtraction point of $\varepsilon=0.5$. 
The phenomenological prediction of Bernauer et al. comes from fits to world form factor data with an assumed functional form for the TPE contribution [27].

OLYMPUS measures $R_{2 \gamma}$ to be somewhat smaller than predicted. The OLYMPUS data are for the most part consistent with unity over much the OLYMPUS acceptance, and in fact fall below unity at low $Q^{2}$ and high $\varepsilon$, which is a prediction of Bernauer (and to a lesser extent, Tomalak). The slope of the data points is better matched by the Blunden calculations.

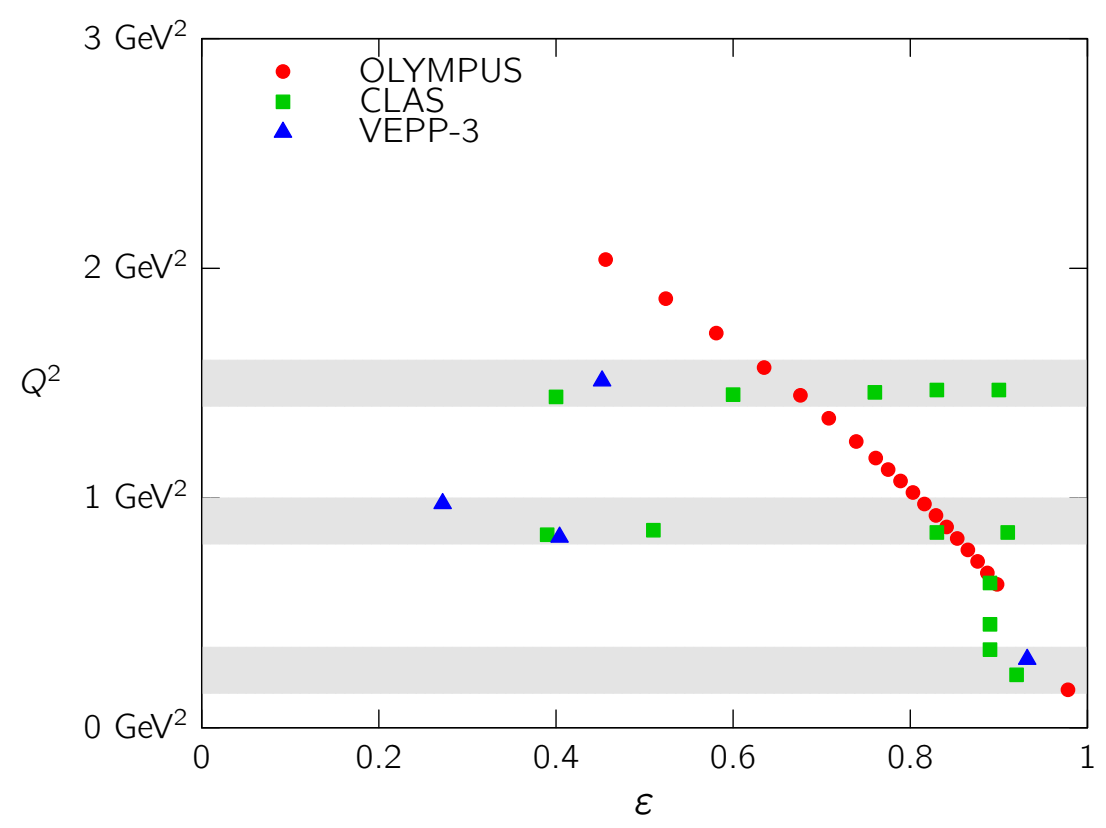

Figure 4: The results of the three recent TPE experiments are difficult to compare to one another because they are situated at different positions on the two-dimensional $\varepsilon, Q^{2}$ kinematic plane.

It is not straight-forward to compare the results of the three TPE experiments, because the kinematics of elastic scattering are two-dimensional. Figure 4 shows the kinematics for the data points of all three experiments in the $\varepsilon, Q^{2}$ plane. There is no reason that data points at similar values of $\varepsilon$ be consistent with each other, if they fall at very different values of $Q^{2}$. To permit a comparison, I show, in figure 5, the data in three bands of similar $Q^{2}$, which are illustrated by gray bars in figure 4. In all three $Q^{2}$ regions, there are no inconsistencies between the three experiments, as seen in figure 5 .

\section{Conclusions}

The results from OLYMPUS neither favor the null hypothesis (no hard TPE) because they show a slope in $R_{2 \gamma}$, nor do they strongly confirm the TPE hypothesis. OLYMPUS measured $R_{2 \gamma}$ to be smaller than most theoretical predictions. This raises the possibility that there may be some unconsidered effects which must be added to the present theory calculations.

The important question that must be asked of the TPE results is whether or not the measured $R_{2 \gamma}$ resolves the form factor discrepancy. In an attempt to answer that question, I developed a phenomenological scheme for estimating the necessary $R_{2 \gamma}$ from global fits to polarized and unpolarized measurements of the proton's form factors (details are described in appendix D of [38]. 


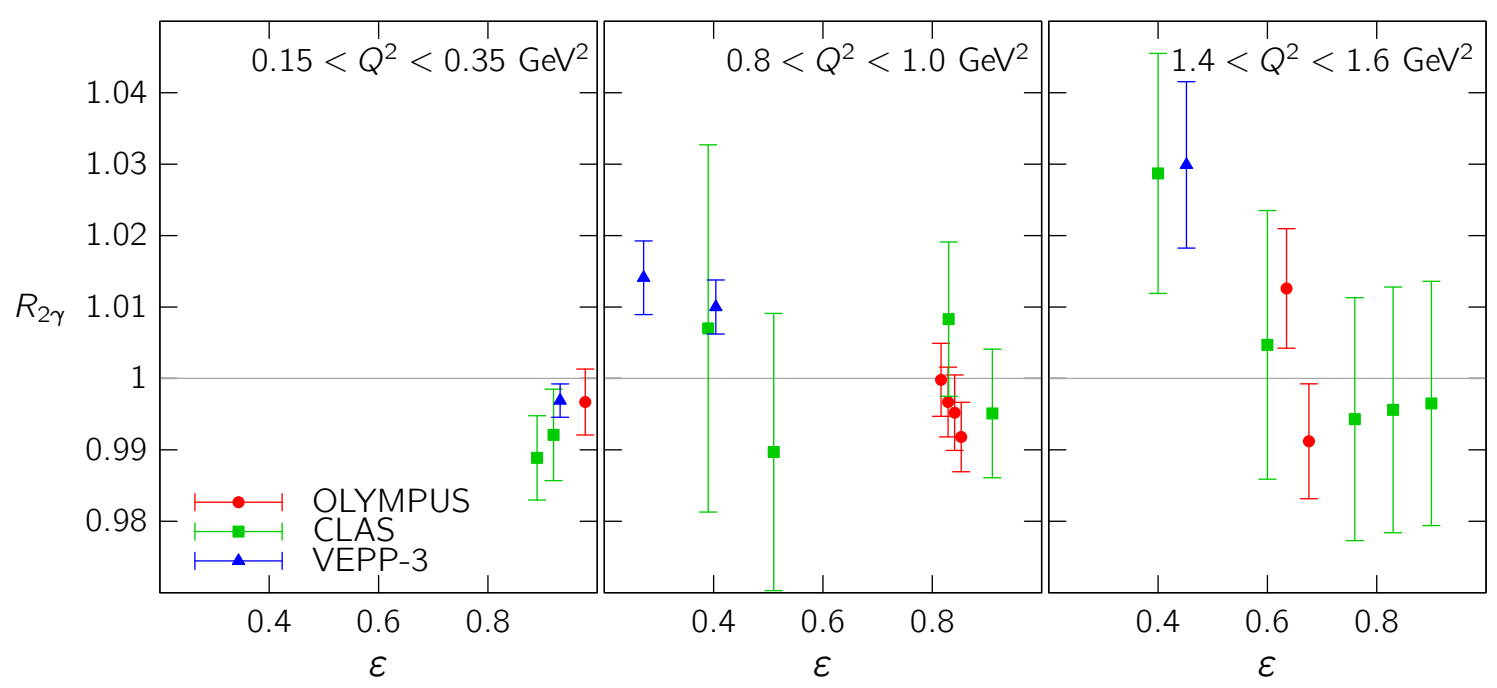

Figure 5: The measurements from the three recent $R_{2 \gamma}$ experiments $[1,34,37]$ are largely consistent with each other.

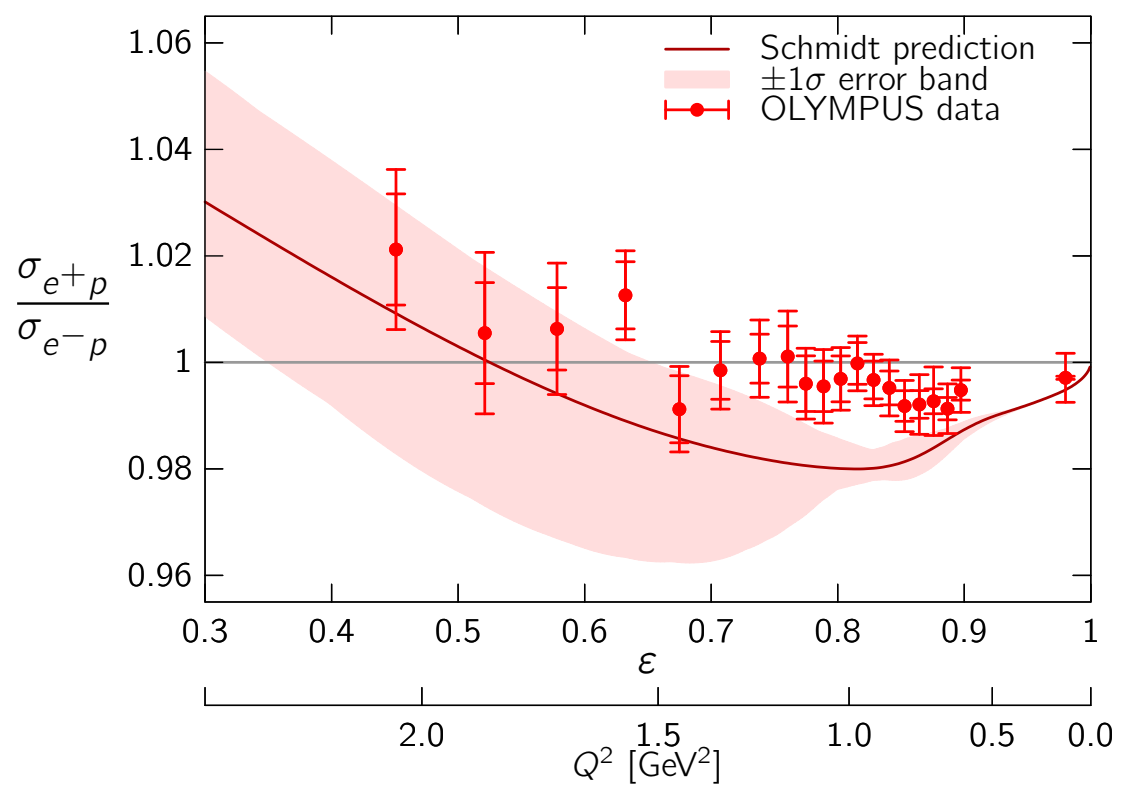

Figure 6: The amount of TPE needed to resolve the form factor discrepancy is not precisely determined, as seen by the uncertainty band from the phenomenological prediction of the author.

Global fits that are published with uncertainties allow an estimate of the uncertainty in the phenomenological prediction. Figure 6 shows the results for OLYMPUS kinematics. The input global fits for unpolarized data are taken from [27], while for the polarized data, the parameterization $\mu_{p} G_{E} / G_{M}=1-0.12 Q^{2} / \mathrm{GeV}^{2}$ was used. The uncertainty band is derived from the quoted uncertainties in [27]. As can be seen from the figure, the OLYMPUS measurement was made in kinematics in which little hard TPE is needed to resolve the discrepancy. Furthermore, the size of the needed TPE effect is not well constrained, especially for $Q^{2}>1 \mathrm{GeV}^{2}$. At these larger values of $Q^{2}$, the OLYMPUS data are fully consistent with the phenomenological prediction, which implies 
that the TPE hypothesis is still completely valid.

For a more definitive test to rule out confirm the TPE hypothesis, a higher momentum transfer (and thus greater beam energy) is needed. At these kinematics, the form factor discrepancy is larger, and so the size of the needed TPE effect is greater, making it easier to distinguish between the hypotheses. However, at higher energy, the elastic ep cross section falls rapidly, making such experiments challenging. While there are no definite proposals for future experiments yet, the community is beginning to discuss options for how a future TPE experiment might be conducted, for example, through the addition of a positron source for the CEBAF accelerator at Jefferson Lab [39]. Though the small elastic cross section will make future experiments lengthy and costly, a measurement of $R_{2 \gamma}$ at $Q^{2}>4 \mathrm{GeV}^{2}$ would allow a decisive test of the TPE hypothesis.

\section{References}

[1] OLYMPUS collaboration, B. S. Henderson et al., Hard Two-Photon Contribution to Elastic Lepton-Proton Scattering: Determined by the OLYMPUS Experiment, Phys. Rev. Lett. 118 (2017) 092501, [1611.04685].

[2] O. Gayou et al., Measurements of the elastic electromagnetic form-factor ratio $\mu_{p} G_{E_{p}} / G_{M_{p}}$ via polarization transfer, Phys. Rev. C64 (2001) 038202.

[3] V. Punjabi et al., Proton elastic form-factor ratios to $Q^{2}=3.5 \mathrm{GeV}^{2}$ by polarization transfer, Phys. Rev. C71 (2005) 055202, [nucl-ex/0501018].

[4] Resonance Spin Structure collaboration, M. K. Jones et al., Proton $G_{E} / G_{M}$ from beam-target asymmetry, Phys. Rev. C74 (2006) 035201, [nucl-ex/ 0606015 ].

[5] A. J. R. Puckett et al., Recoil Polarization Measurements of the Proton Electromagnetic Form Factor Ratio to $Q^{2}=8.5 \mathrm{GeV}^{2}$, Phys. Rev. Lett. 104 (2010) 242301, [nucl-ex/1005.3419].

[6] M. Paolone et al., Polarization Transfer in the ${ }^{4} \mathrm{He}\left(e, e^{\prime} p\right)^{3} \mathrm{H}$ Reaction at $Q^{2}=0.8$ and $1.3(\mathrm{GeV} / \mathrm{c})^{2}$, Phys. Rev. Lett. 105 (2010) 072001, [1002.2188].

[7] A. J. R. Puckett et al., Final Analysis of Proton Form Factor Ratio Data at $Q^{2}=4.0,4.8$ and 5.6 GeV', Phys. Rev. C85 (2012) 045203, [nucl-ex/1102.5737].

[8] J. Litt et al., Measurement of the ratio of the proton form-factors, $g_{e} / g_{m}$, at high momentum transfers and the question of scaling, Phys. Lett. B31 (1970) 40-44.

[9] W. Bartel, F. W. Busser, W. r. Dix, R. Felst, D. Harms, H. Krehbiel et al., Measurement of proton and neutron electromagnetic form-factors at squared four momentum transfers up to $3 \mathrm{GeV} / \mathrm{c}^{2}$, Nucl. Phys. B58 (1973) 429-475.

[10] L. Andivahis et al., Measurements of the electric and magnetic form-factors of the proton from $Q^{2}=1.75$ to $8.83(\mathrm{GeV} / \mathrm{c})^{2}$, Phys. Rev. D50 (1994) 5491-5517.

[11] R. C. Walker et al., Measurements of the proton elastic form-factors for $1 \leq Q^{2} \leq 3(\mathrm{GeV} / \mathrm{c})^{2}$ at SLAC, Phys. Rev. D49 (1994) 5671-5689.

[12] E94110 collaboration, M. E. Christy et al., Measurements of electron proton elastic cross-sections for $0.4<Q^{2}<5.5(\mathrm{GeV} / \mathrm{c})^{2}$, Phys. Rev. C70 (2004) 015206, [nucl-ex/ 0401030 ].

[13] I. A. Qattan et al., Precision Rosenbluth measurement of the proton elastic form-factors, Phys. Rev. Lett. 94 (2005) 142301, [nucl-ex/ 0410010 ]. 
[14] M. N. Rosenbluth, High Energy Elastic Scattering of Electrons on Protons, Phys. Rev. 79 (1950) 615-619.

[15] BATES FPP collaboration, B. D. Milbrath et al., A Comparison of polarization observables in electron scattering from the proton and deuteron, Phys. Rev. Lett. 80 (1998) 452-455, [nucl-ex/ 9712006 ].

[16] L. W. Mo and Y.-S. Tsai, Radiative corrections to elastic and inelastic ep and $v p$ scattering, Rev. Mod. Phys. 41 (Jan, 1969) 205-235.

[17] L. C. Maximon and J. A. Tjon, Radiative corrections to electron-proton scattering, Phys. Rev. C 62 (Oct, 2000) 054320, [nucl-th/ 0002058 ].

[18] P. Blunden, W. Melnitchouk and J. Tjon, Two-photon exchange in elastic electron-nucleon scattering, Phys.Rev. C72 (2005) 034612, [nucl-th/ 0506039 ].

[19] S. Kondratyuk, P. Blunden, W. Melnitchouk and J. Tjon, Delta resonance contribution to two-photon exchange in electron-proton scattering, Phys.Rev.Lett. 95 (2005) 172503, [nucl-th/ 0506026 ].

[20] S. Kondratyuk and P. Blunden, Contribution of spin $1 / 2$ and $3 / 2$ resonances to two-photon exchange effects in elastic electron-proton scattering, Phys.Rev. C75 (2007) 038201, [nucl-th/ 0701003 ].

[21] D. Borisyuk and A. Kobushkin, Two-photon-exchange amplitude with $\pi N$ intermediate states: $P_{33}$ channel, Phys.Rev. C89 (2014) 025204, [hep-ph/1306.4951].

[22] O. Tomalak and M. Vanderhaeghen, Subtracted dispersion relation formalism for the two-photon exchange correction to elastic electron-proton scattering: comparison with data, Eur. Phys. J. A51 (2015) 24, [1408.5330].

[23] P. G. Blunden and W. Melnitchouk, A dispersive approach to two-photon exchange in elastic electron-proton scattering, 1703.06181.

[24] A. V. Afanasev, S. J. Brodsky, C. E. Carlson, Y.-C. Chen and M. Vanderhaeghen, The Two-photon exchange contribution to elastic electron-nucleon scattering at large momentum transfer, Phys.Rev. D72 (2005) 013008, [hep-ph/ 0502013$].$

[25] Y. C. Chen, C. W. Kao and S. N. Yang, Is there model-independent evidence of the two-photon-exchange effect in the electron-proton elastic scattering cross section?, Phys.Lett. $\mathbf{B} 652$ (2007) 269-274, [nucl-th/0703017].

[26] Guttmann, Julia and Kivel, Nikolai and Meziane, Mehdi and Vanderhaeghen, Marc, Determination of two-photon exchange amplitudes from elastic electron-proton scattering data, Eur.Phys.J. A47 (2011) 77, [hep-ph/1012.0564].

[27] A1 collaboration, J. C. Bernauer et al., Electric and magnetic form factors of the proton, Phys. Rev. C90 (2014) 015206, [nucl-ex/1307.6227].

[28] Y. Bystritskiy, E. Kuraev and E. Tomasi-Gustafsson, Structure function method applied to polarized and unpolarized electron-proton scattering: A solution of the $G E(p) / G M(p)$ discrepancy, Phys.Rev. C75 (2007) 015207, [hep-ph/ 0603132$].$

[29] E. A. Kuraev, V. V. Bytev, S. Bakmaev and E. Tomasi-Gustafsson, Charge asymmetry for electron (positron)-proton elastic scattering at large angle, Phys. Rev. C78 (2008) 015205, [0710.3699].

[30] OLYMPUS collaboration, R. Milner et al., The OLYMPUS Experiment, Nucl. Instrum. Meth. A741 (2014) 1-17, [1312.1730].

[31] J. C. Bernauer, V. Carassiti, G. Ciullo, B. S. Henderson, E. Ihloff, J. Kelsey et al., The OLYMPUS Internal Hydrogen Target, Nucl. Instrum. Meth. A755 (2014) 20-27, [14 04 . 0579]. 
[32] D. Hasell et al., The BLAST experiment, Nucl. Instrum. Meth. A603 (2009) 247-262.

[33] Pérez Benito, R. and Khaneft, Dmitry and O'Connor, Colton and Capozza, Luigi and Diefenbach, Jürgen and Gläser, Boris and Ma, Yue and Maas, Frank E. and Rodríguez Piñeiro, D., Design and Performance of a Lead Fluoride Detector as a Luminosity Monitor, Nucl. Instrum. Meth. A826 (2016) 6-14, [1602.01702].

[34] I. A. Rachek et al., Measurement of the two-photon exchange contribution to the elastic $e^{ \pm} p$ scattering cross sections at the VEPP-3 storage ring, Phys. Rev. Lett. 114 (2015) 062005, [1411. 7372 ].

[35] CLAS collaboration, M. Moteabbed et al., Demonstration of a novel technique to measure two-photon exchange effects in elastic $e^{ \pm}$p scattering, Phys. Rev. C88 (2013) 025210, [1306.2286].

[36] CLAS collaboration, D. Adikaram et al., Towards a resolution of the proton form factor problem: new electron and positron scattering data, Phys. Rev. Lett. 114 (2015) 062003, [1411.6908].

[37] CLAS collaboration, D. Rimal et al., Measurement of two-photon exchange effect by comparing elastic $e^{ \pm}$p cross sections, Phys. Rev. C95 (2017) 065201, [1603.00315].

[38] A. Schmidt, Measuring the lepton sign asymmetry in elastic electron-proton scattering with OLYMPUS. PhD thesis, Massachusetts Institute of Technology, Cambridge, Massachusetts, 2016.

[39] E. Voutier, Physics potential of polarized positrons at the Jefferson Laboratory, Nucl. Theor. 33 (2014) 142-151, [1412.1249]. 